\title{
Efecto de la sulfidez en la respuesta a flexión de tableros de cemento reforzados con fibras de Guadua angustifolia kunth pre-tratadas alcalinamente
}

\author{
Effects of sulphidity on flexural strength of fiber-cement boards reinforced \\ with guadua angustifolia kunth fibers with alkaline pretreatments
}

\author{
Luz Adriana Sanchez-Echeverri ${ }^{1,2}{ }^{2} \quad$ Jorge Alberto Medina-Perilla ${ }^{1}$ \\ Germán Camilo Quintana $^{3} \quad$ Eshmaiel Ganjian ${ }^{4}$
}

Recibido 20 de junio de 2019, Aceptado 12 de mayo de 2020

Received: June 20, 2019 Accepted: May 12, 2020

\begin{abstract}
RESUMEN
La Guadua angustifolia Kunth (GAK) es una bamboo nativo del continente americano con gran adaptabilidad ecológica. Su composición y estructura la convierte en una fuente lignocelulósica de buenas propiedades mecánicas. En este trabajo, fibras de $G A K$ fueron pre-tratadas alcalinamente, mediante pulpeo Kraft a baja temperatura, se fijó un valor de álcali efectivo del $10 \%$ combinado con tres valores de sulfidez $10 \%, 15 \%$ y $30 \%$. Se evaluaron los cambios en la superficie de la fibra y el valor en la retención de agua después de los tratamientos alcalinos. Con una porción en masa del $5 \%$ de fibras pre-tratadas se fabricaron tableros de cemento reforzados y se evaluó los efectos de la concentración de la sulfidez del tratamiento alcalino en las propiedades físico-mecánicas de los tableros. Los tableros de cemento reforzados con fibras fueron evaluados mecánicamente a los 7, 28 y 180 días de fraguado. Los resultados mostraron que la resistencia a la flexión en tableros reforzados con fibras tratadas es mayor que en tableros reforzados con fibras no tratadas. Después de 180 días de fraguado no se observó reducción de la resistencia a flexión lo que indica que los tratamientos alcalinos evitaron la degradación de las fibras en la matriz de cemento. Finalmente, los resultados mostraron que un valor menor en la sulfidez (10\%) conduce a fibras con superficies más limpias mejorando la adherencia en la matriz fibra-cemento y produciendo tableros compuestos de cemento y G. angustifolia Kunth con mayor desempeño mecánico.
\end{abstract}

Palabras clave: Compuesto fibra-cemento, propiedades físico-mecánicas, fibras naturales, tratamiento alcalino.

\section{ABSTRACT}

Guadua angustifolia KUNTH is a giant grass native to America with high ecological adaptability. Its composition and structure make it in a lignocellulosic source with good mechanical properties with wide applications. Guadua angustifolia fibers were alkali pretreated by Kraft pulping at low temperatures. An amount of $10 \%$ of active alkali was combined with three different sulphide levels - 10\% - 15\%, and 30\%. A

1 Universidad de los Andes. Departamento de Ingeniería Mecánica. Bogotá, Colombia.

E-mail: se.luz10@uniandes.edu.co; jmedina@uniandes.edu.co

2 Universidad de Ibagué. Facultad de Ciencias Naturales y Matemáticas. Ibagué, Colombia.

E-mail: luz.sanchez@unibague.edu.co

3 Universidad Pontificia Bolivariana. Facultad de Ingeniería Química. Grupo Pulpa y Papel. Medellín, Colombia. E-mail: german.quintana@upb.edu.co

4 Coventry University. Department of Civil Engineering, Architecture and Building. Coventry, United Kingdom. E-mail: cbx111@ coventry.ac.uk

* Autor de correspondencia: se.luz10@uniandes.edu.co; luz.sanchez@unibague.edu.co. 
mass portion of $5 \%$ of pretreated fibers was used to reinforce the fiber cement board composites produced by the slurry method. The effects of sulfidity concentration in treatment over the physical-mechanical properties of fiber cement board composites were evaluated. Superficial changes on Guadua fiber and water retention value after alkaline treatments were determined. Fiber cement boards were mechanically tested after 7, 28, and 180 days of curing. Results showed flexural strength in boards reinforced with treated fibers is greater than flexural strength in boards reinforced with untreated fibers; moreover, after 180 days of curing, there was no flexural strength reduction, indicating that alkaline treatments avoid fiber degradation into the cement matrix. Finally, results showed that low sulfidity concentration (10\%) produces fibers with cleaner surfaces, improving fiber-cement bonding, and these fibers allowed the production of fiber cement boards with higher flexural strength.

Keywords: Fiber-cement composite, mechanic and physic properties, natural fibers, alkali treatment.

\section{INTRODUCCIÓN}

El desarrollo social y económico de los países está ligado estrechamente con la mejor utilización de los recursos disponibles con el fin de introducirlos en nuevos productos con un valor agregado. Los materiales compuestos de fibrocementos desarrollados con fibras vegetales son conocidos por ser eco-amigables, de bajo costo y por exhibir mejores propiedades mecánicas comparadas con otros compuestos de cemento [1, $2,3]$. En países en desarrollo donde se requieren productos de construcción de bajo costo, el uso de las fibras vegetales no madereras como reemplazo de los peligrosos asbestos, fibras de vidrio e incluso fibras de madera en compuestos de cemento y concreto es una alternativa viable. Para la fabricación de tableros compuestos de cemento se han utilizado diferentes tipos de fibras naturales como coco, jute, eucalipto entre otras $[4,5,6]$; propias de los sitios de origen de los diferentes autores. La mayoría de trabajos en refuerzo de matrices de cemento, utilizan fibras de jute, coco y eucalipto $[2,7,8,9]$; sin embargo, no existen muchos trabajos en los que se hayan utilizado fibras provenientes de recursos con alta abundancia en Colombia, como es el caso de las extraídas de Guadua angustifolia Kunth. En Colombia existen alrededor de 55000 Hectáreas plantadas donde el $95 \%$ y $97 \%$ de los cultivos son naturales, no requieren mantenimiento y han crecido por características de suelo y clima de las regiones. Esta ventaja sumada a sus buenas propiedades mecánicas hace que sus fibras se conviertan en una fuente lignocelulósica adecuada para la elaboración de materiales compuestos.

La efectividad de las fibras para mejorar las propiedades mecánicas de los tableros de cemento depende de las interacciones fibra-matriz y en particular de la interface que se origina y de su capacidad para trasmitir los esfuerzos generados $[6,10,11,12]$. Las propiedades de la interfaz se pueden mejorar modificando la matriz de cemento $[13,14]$ o modificando la superficie de las fibras con la formación de capas protectoras que puedan incrementar la compatibilidad y adherencia a la matriz de cemento $[5,15,16]$. La modificación de las fibras se consigue por medio de pretratamientos que incluyen métodos químicos, físicos y fisicoquímicos. Dentro de los pretratamientos químicos más exitosos en la modificación de la superficie y deslignificación de estos materiales se encuentran los tratamientos alcalinos; estos tratamientos han mostrado tener una buena efectividad segregando las fibras, despolimerizando y condensando la lignina e hidrolizando las hemicelulosas [17] lo que mejora la respuesta mecánica de los compuestos de cemento.

El pulpeo Kraft, método alcalino utilizado en la industria del papel para obtener celulosa de materiales madereros y no madereros, es una alternativa que permite remover compuestos no-celulósicos de los materiales lignocelulósicos sin degradar los carbohidratos estructurales como la celulosa. Los tableros compuestos de cemento reforzados con pulpa de fibra Kraft han mostrado un mejoramiento en la respuesta a flexión $[12,18]$.

En el pulpeo Kraft, las propiedades finales de la pulpa dependen de la sulfidez, de la temperatura, del álcali activo, de la razón licor/fibra y del tiempo de cocción. Dentro de estas variables se ha mostrado que la sulfidez y el álcali efectivo tienen una mayor influencia en el rendimiento del pulpeo Kraft así como en el grado de delignificación de la pulpa [19]. En este trabajo se obtuvieron pulpas de Guadua 
angustifolia Kunth por medio del pretratamiento alcalino Kraft a bajas condiciones de cocción. Para el tratamiento alcalino se empleó un álcali activo del $10 \%$ y una variación de tres niveles de sulfidez: $10 \%, 15 \%$ y $30 \%$. Las fibras pre-tratadas se utilizaron como refuerzo en la elaboración de tableros compuestos de cemento. Estos tableros fueron evaluados a $\operatorname{los} 7,28$ y 180 días de curado mediante un test de flexión a 3 puntos con el objetivo de determinar el efecto de la variación de la sulfidez en la respuesta a flexión.

\section{MATERIALES Y MÉTODOS}

\section{Materiales}

Las fibras de G. angustifolia Kunth fueron obtenidas de internodos provenientes de la basa del tallo o culmo, dado que es la parte más usada en Colombia para fines de construcción. Se utilizaron culmos con 3 años de siembra con un espesor de pared promedio de $20 \mathrm{~mm}$ y un diámetro promedio de $12 \mathrm{~mm}$. Los culmos fueron obtenidos de un campo experimental ubicado en la Tebaida Quindío-Colombia (Longitud: O75 $47^{\prime} 14,86^{\prime \prime}$ Latitud: N4²7'9.54") con una temperatura promedio de $24^{\circ} \mathrm{C}$. Para la fabricación de los compuestos se utilizó cemento Portland Tipo I (CP) según la norma BS EN 197-1: 2000 (Cement. Composition, specifications and conformity criteria for common cements) con un área superficial específica de $2600 \mathrm{~cm}^{2} \mathrm{~g}^{-1}$ y peso específico de 3-3,25 ton $\mathrm{m}^{-3}$. Para el pulpeo Kraft se utilizaron lentejas de Hidróxido de Sodio $(\mathrm{NaOH})$ y escamas de Sulfuro de Sodio $\left(\mathrm{Na}_{2} \mathrm{~S}\right)$ con una solubilidad en agua a $20^{\circ} \mathrm{C}$ de $1,09 \mathrm{~g} / \mathrm{L} \mathrm{y} 187 \mathrm{~g} / \mathrm{L}$ respectivamente.

\section{Pre-tratamiento alcalino}

El pretratamiento alcalino busca la desintegración de la guada mediante la disolución de la lignina en presencia de álcali y alta temperatura. De los internodos de la G. angustifolia Kunth se cortaron tiras con dimensiones de $20 \mathrm{~mm} \times 50 \mathrm{~mm} \times 30 \mathrm{~mm}$ para ser utilizadas en el pretratamiento alcalino. El pretratamiento alcalino empleado en este trabajo mantuvo fijo un álcali activo de $10 \%$ con una combinación de 3 niveles de sulfidez (10\%, 15\% y $30 \%$ ). La Tabla 1 muestra los valores de hidróxido de sodio y sulfuro de sodio utilizados para la elaboración del licor blanco para el pretratamiento de las fibras.

Las fibras fueron sumergidas en el licor blanco $\left(\mathrm{NaOH}+\mathrm{Na}_{2} \mathrm{~S}\right)$ en una relación 5:1, las fibras en el licor se auto clavaron en un autoclave eléctrico All American a una temperatura de $125^{\circ} \mathrm{C} \pm 2{ }^{\circ} \mathrm{C}$ y una presión de $1,25 \mathrm{KPa}$ durante $2 \mathrm{~h}$. Después de la cocción, las fibras se lavaron con abundante agua de grifo durante 15 min con el fin de garantizar la remoción de exceso de reactivos que no reaccionaron. Finalmente, las fibras se secaron a temperatura ambiente durante $24 \mathrm{~h}$. La Figura 1 muestra el procedimiento para obtener las fibras de Guadua angustifolia Kunth y las fibras obtenidas usadas como refuerzo en la fabricación del compuesto.

\section{Morfología de las fibras}

Para determinar el efecto de del pre-tratamiento alcalino sobre la longitud y espesor de las fibras de

Tabla 1. Valores de Hidróxido de Sodio y Sulfuro de Sodio para la elaboración del licor blanco usado para el pretratamiento de las fibras de G. angustifolia Kunth.

\begin{tabular}{|c|c|c|c|}
\hline $\begin{array}{c}\text { Sulfidez } \\
{[\%]}\end{array}$ & $\begin{array}{c}\text { G. angustifolia } \\
\text { Kunth }[\mathbf{g}]\end{array}$ & $\begin{array}{c}\mathbf{N a O H} \\
{[\mathbf{g}]}\end{array}$ & $\begin{array}{c}\mathbf{N a}_{2} \mathbf{S} \\
{[\mathbf{g}]}\end{array}$ \\
\hline 30 & 100 & 9,00 & 3,80 \\
\hline 15 & 100 & 10,97 & 1,89 \\
\hline 10 & 100 & 11,62 & 1,25 \\
\hline
\end{tabular}

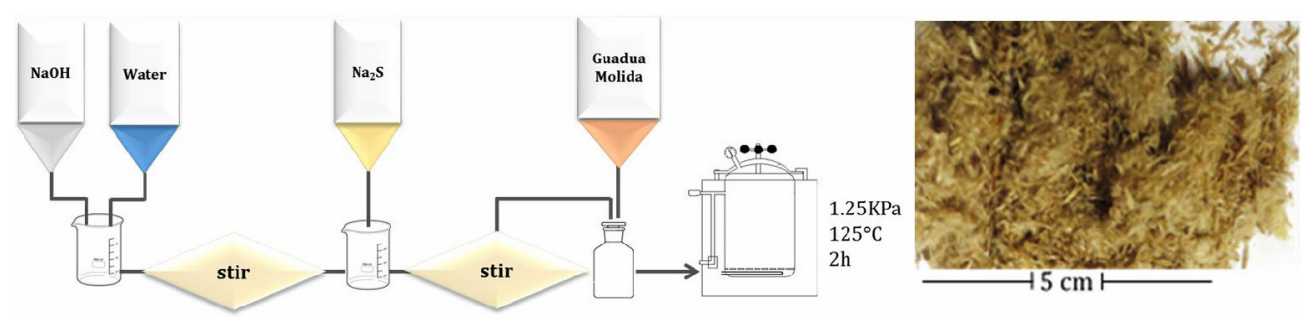

Figura 1. (Izquierda) Procedimiento para la obtención de fibra de Guadua angustifolia Kunth. (Derecha) Fibra de Guadua angustifolia Kunth usada como refuerzo en la elaboración de tableros de cemento. 
Guadua éstas fueron analizadas en un microscopio óptico. Las imágenes obtenidas fueron procesadas mediante el software libre ImageJ donde se tomó la longitud y espesor de 100 fibras para obtener una longitud y espesor promedio de las fibras antes y después del tratamiento.

La morfología y superficie de las fibras antes y después del tratamiento fue evaluada en un microscopio electrónico de barrido marca JEOL JSM-6490LV en alto vacío y un voltaje de 20-25 KV. Las fibras fueron recubiertas por una capa de grafito para garantizar la conductividad eléctrica. Diferentes amplificaciones se utilizaron para la toma de micrografías.

\section{Retención de agua}

Para evaluar el efecto del tratamiento alcalino en la retención de agua de las fibras se determinó el valor de retención de agua o WRV por sus siglas en inglés. Se colocó una almohadilla de fibras de aproximadamente $5 \mathrm{~g}$ previamente humedecidas durante $1 \mathrm{~h}$, y posteriormente, se centrifugaron a una velocidad de $3000 \mathrm{rpm}$ en una centrífuga marca Boeco U-320R durante 15 min para eliminar el agua de las superficies exteriores y el agua de los lúmenes de las fibras. Después de este tiempo se retiran las almohadillas y se secan en un horno convencional a $105^{\circ} \mathrm{C} \pm 2{ }^{\circ} \mathrm{C}$ durante $24 \mathrm{~h}$. Finalmente se pesan para calcular el WRV por medio de la ecuación (1).

$$
\begin{gathered}
\text { Retención de Agua } \\
\mathrm{WRV}=\mathrm{W} \text { humedo }-\mathrm{W} \text { secoW humedo } \times 100 \%
\end{gathered}
$$

Donde $\mathrm{W}_{\text {humedo }}$ indica el peso de la almohadilla después de la centrifugación y $\mathrm{W}_{\text {seco }}$ el peso de la almohadilla después de $24 \mathrm{~h}$ de secado.

\section{Drenabilidad (freeness)}

La drenabilidad de una pulpa es un fenómeno que se presenta inter-fibras y suministra una medida de la velocidad a la cual drena una suspensión de pulpa bajo condiciones normalizadas; esta medida se encuentra asociada con fracción de finos de la muestra los cuales se pueden producir después de tratamientos químicos o mecánicos. Se empleó la norma Tappi T227-1999 (Freeness of pulp - Canadian Standard Freeness (CSF)) para determinar la drenabilidad de las fibras antes y después del tratamiento alcalino. El valor del CSF medido en mililitros se determinó por triplicado a partir de una suspensión de muestra previamente desintegrada con una consistencia de $0,3 \%$ ( $3 \mathrm{~g}$ de fibras en $1 \mathrm{~L}$ de agua) como lo indica la norma.

\section{Preparación de tableros compuestos de cementos} Para la fabricación de los tableros compuestos de cemento se seleccionó una fracción de peso seco de $5 \%$ de fibra pre-tratada alcalinamente; el porcentaje de fibra se determinó de acuerdo a los reportes de la literatura los cuales muestran que el porcentaje de fibra que presenta mejores características mecánicas está entre 3\%-8\%. Para la manufactura de los tableros compuestos de cemento se utilizaron $450 \mathrm{ml}$ de agua y $130 \mathrm{~g}$ de cemento portland. Se elaboraron 9 muestras por cada uno de los pretratamientos. 3 muestras fueron ensayadas a $\operatorname{los}$ 7, 28 y 180 días de curado. Para efectos de comparación se elaboraron muestras de control con $5 \%$ de fibra no tratada las cuales también fueron ensayadas a los 7, 28 y 180 días de curado. Los Tableros fueron elaborados en un molde de $180 \mathrm{~mm}$ x $8 \mathrm{~mm}$, el espesor de los tableros fue en promedio $6,5 \mathrm{~mm}$; la presión utilizada para la fabricación de los tableros fue de $100 \mathrm{MPa}$. La Figura 2 muestra el proceso de manufactura de los tableros compuestos de cemento.

Después de su elaboración, los tableros se pesaron para determinar la nueva relación másica agua/ cemento (W/C). El curado de las muestras se llevó a temperatura ambiente $\left(20 \pm 1^{\circ} \mathrm{C}\right)$ y humedad relativa $(65 \% \pm 1 \%)$ controladas durante 7, 28 y 180 días antes de las pruebas mecánicas. Las muestras fueron etiquetadas como KA10SX, donde K indica pretratamiento alcalino, A10 álcali activo 10\% y SX determina el grado de sulfidez. Para las muestras no tratadas se utilizó la etiqueta "No Tratada" y se le asigna una sulfidez de cero.

\section{Respuesta Mecánica}

Los tableros se ensayaron a flexión de tres puntos por triplicado, de acuerdo al estándar BS EN 12467:2012 (Fibre-cement flat sheets - Product specification and test methods). Los ensayos se realizaron después de 7, 28 y 180 días de curado en una máquina de ensayos JJ Lloyd conectada a un sistema de adquisición de datos con el software Noxygen. La velocidad del ensayo fue de $5 \mathrm{~mm} /$ min y una distancia entre apoyos de $150 \mathrm{~mm}$. Con los datos obtenidos se calculó el módulo de ruptura (MOR) en seco para todos los tableros. 


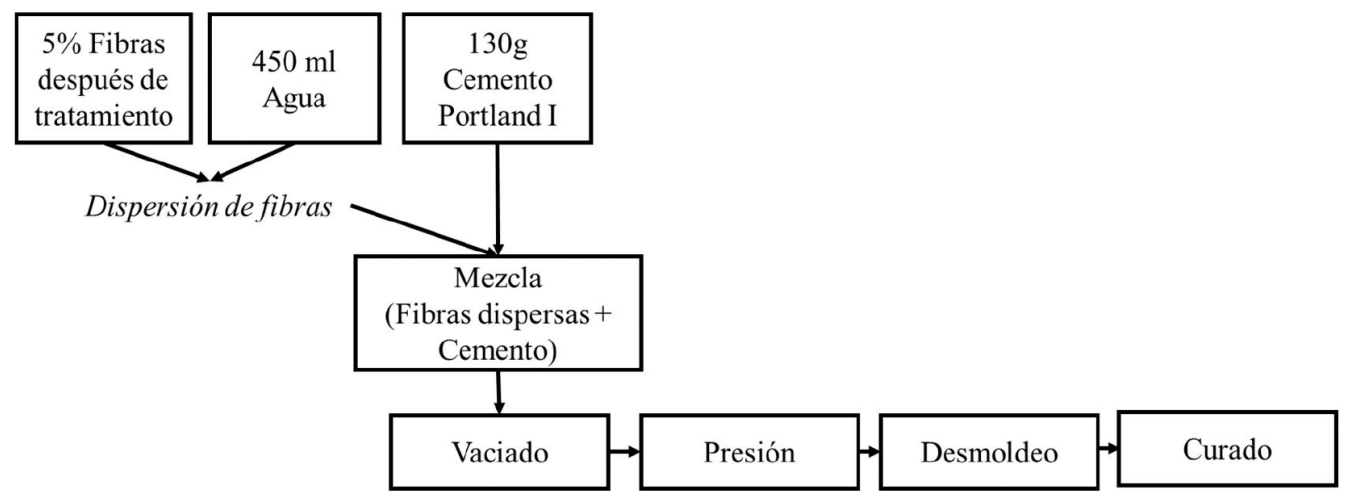

Figura 2. Proceso para la fabricación de tableros de cemento reforzados con Guadua angustifolia Kunth.

\section{Análisis de covarianza}

Dado que el valor de la relación agua/cemento (W/C) tiene un efecto sobre la resistencia a la flexión en placas de fibrocemento, a los resultados obtenidos se les realizó un Análisis de Covarianza con el fin de poder independizar la respuesta de la resistencia a la flexión del valor de la relación W/C. El análisis de covarianza es una técnica que combina el Análisis de Regresión con el Análisis de Varianza. Cuando existe covarianza se observa la variación simultánea de dos variables que se asume están influyendo sobre la variable respuesta [20]. Para comparar los efectos de los factores sobre la variable respuesta se calcularon sus respectivos intervalos de confianza basados en el procedimiento de la diferencia mínima significativa (LSD) de Fisher. Estos intervalos están construidos de tal manera que, si dos medias son iguales, sus intervalos se traslaparán. Cualquier par de intervalos que no se traslapen verticalmente corresponden a pares de medias que tienen una diferencia estadísticamente significativa. Para este análisis se estudió el efecto de dos factores: sulfidez del tratamiento alcalino y el tiempo de curado sobre la resistencia a la flexión teniendo el valor de W/C como covariable. Para los tableros de cemento reforzados con fibras sin tratamiento alcalino se le asignó un valor de sulfidez de cero.

\section{Caracterización superficies de fractura}

Para observar la superficie de fractura y las interfaces fibra-matriz en los tableros compuestos de cemento después de la prueba de flexión, se utilizó un microscopio electrónico de barrido (MEB-JEOL JSM-6490LV) en modo de bajo vacío. Antes de la observación MEB las muestras fueron secadas y recubiertas con una capa de grafito para garantizar su conductividad eléctrica.

\section{RESULTADOS Y DISCUSIÓN}

\section{Morfología de las fibras}

La Figura 3 muestra las imágenes del microscopio óptico de las fibras de Guadua angustifolia Kunth antes del tratamiento y después del pre-tratamiento
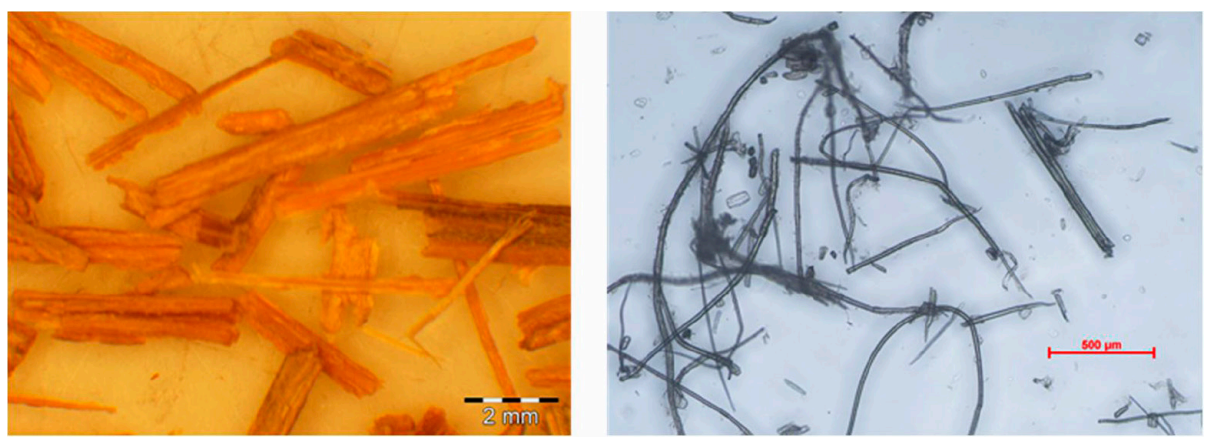

Figura 3. Imágenes de microscopía óptica de fibras G. angustifolia Kunth a) fibras sin tratamiento b) fibras después del pre-tratamiento alcalino. 
alcalino. El espesor y longitud de las fibras fueron calculados mediante el software libre imageJ. Para las fibras no tratadas se obtuvo una longitud promedio de 3,9 $\pm 1,2 \mathrm{~mm}$ con un espesor promedio de $0,615 \pm 0,24 \mathrm{~mm}$; después de aplicarse el tratamiento alcalino las fibras resultantes presentan una longitud promedio de $0,845 \pm 0,2 \mathrm{~mm}$ y un diámetro promedio de 0,0245 $\pm 0,006 \mathrm{~mm}$.

La Figura 4 muestra las imágenes del MEB de las fibras después del tratamiento alcalino, así como de las fibras no tratadas. Las fibras no tratadas no son dispersadas y permanecen como haces de fibras con un espesor alrededor de 0,25 mm (Figura 4d)), mientras que el pretratamiento alcalino permite aislar fibras individuales de estos haces con espesores alrededor de 0,025 mm (Figuras 4a), 4b) y 4c)). Las imágenes de las fibras no tratadas alcalinamente se tomaron en amplificaciones menores a las de las fibras tratadas ya que al no estar dispersadas es necesario una amplificación menor para observar la totalidad de la fibra. Por comparación de la superficie de las fibras no tratadas (Figura (4d)) con la superficie de las fibras pre-tratadas alcalinamente (Figuras (4a), (4b) y (4c)) se observa como el tratamiento alcalino remueve de la superficie algunos compuestos generando superficies más lisas. El pre-tratamiento alcalino logra desintegrar los haces de fibra produciendo más área superficial de reacción en la matriz de cemento.
El obtener una superficie de fibra más lisa y haces desintegrados después del pre-tratamiento alcalino indica que el pre-tratamiento remueve cierta cantidad de lignina, hemicelulosa, varios extractivos (taninos, aceites y ceras) que recubren la parte superficial de las fibras. La remoción de estos compuestos no celulósicos permite que las características adhesivas de las fibras mejoren lo que se traduce en un anclaje mecánico mayor cuando se encuentran dentro de la matriz de cemento.

Las imágenes en los recuadros de la Figura 4 se tomaron con una amplificación de 5000X, estas imágenes muestran los agregados de macrofibrilas de celulosa en las fibras pre-tratadas alcalinamente (Figuras (4a), (4b) y (4c)); mientras que en las fibras sin tratamiento este recuadro muestra algunos compuestos sobre la superficie corroborando que los pre-tratamientos alcalinos remueven estas impurezas de la superficie. Los agregados de microfibrilas de celulosa (fibrilación) aumentan a medida que la sulfidez del tratamiento aumenta según se observa en las figuras.

\section{Retención de agua}

El WRV indica la capacidad que tienen las fibras para absorber agua e hincharse, esta medida se relaciona con la flexibilidad de la fibra y con la fibrilación interna de la pulpa. La Figura 5 muestra el Análisis de Varianza del WRV para las fibras no tratadas y para las fibras
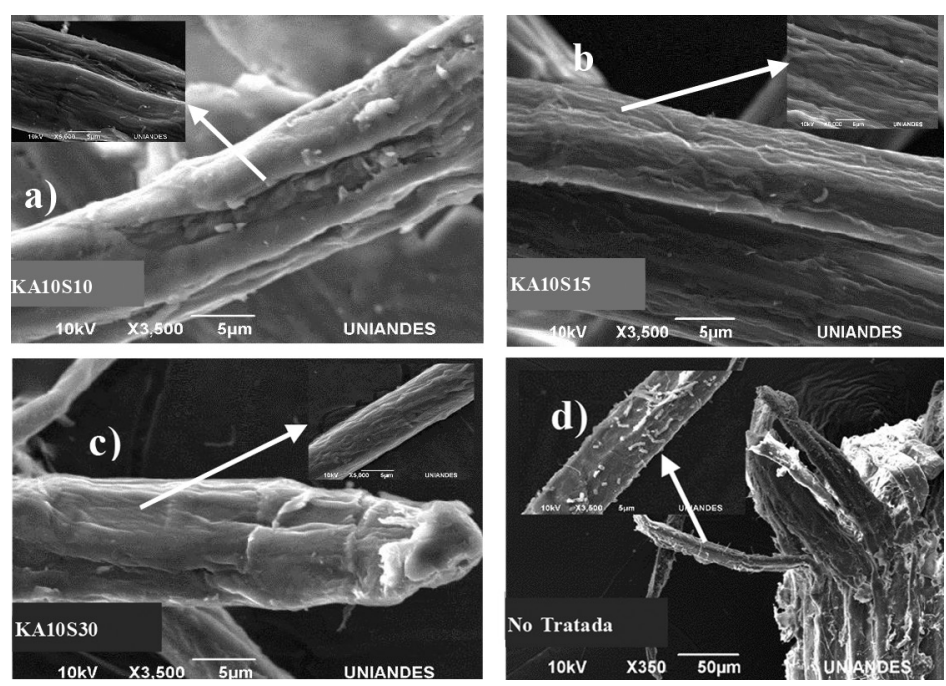

Figura 4. Imágenes MEB de fibras de G. angustifolia Kunth obtenidas mediante pulpeo Kraft con diferente sulfidez: a) 10\%, b) $15 \%$, c) $30 \%$, d) Sin pre-tratamiento. 


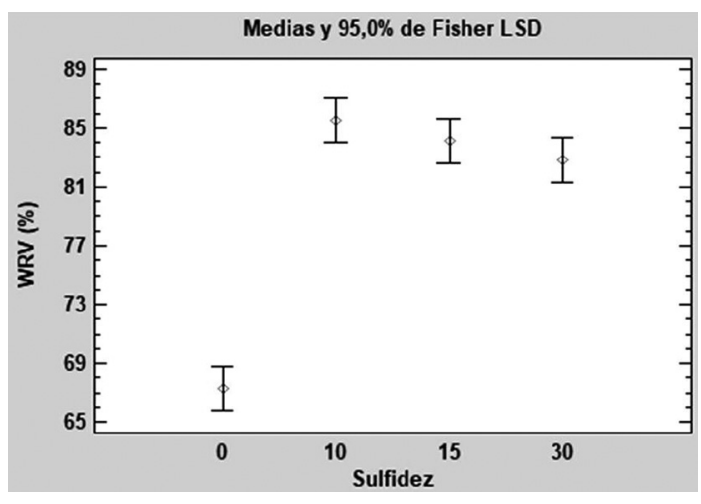

Figura 5. ANOVA para la retención de agua (WRV) de las fibras control y las fibras obtenidas después del pretratamiento alcalino.

obtenidas después del pre-tratamiento alcalino. Es posible ver que después del pre-tratamiento todas las pulpas aumentan su capacidad de retención de agua debido a que los tratamientos alcalinos remueven compuestos no celulósicos lo que aumenta el carácter hidrófilo de la fibra.

Basado en los resultados obtenidos se puede observar que el cambio en la sulfidez no afecta de manera significativa el valor de retención de agua de las fibras; estos hallazgos son diferentes a los resultados reportados previamente por Ponni et al., (2014). Sin embargo, la temperatura a la que se realizó el pre-tratamiento alcalino en este trabajo es inferior a la reportada por estos autores [21]. Esta diferencia en la temperatura crea diferencias sobre los grupos funcionales superficiales y a su vez modifican la capacidad para retener agua.

\section{Drenabilidad (freeness)}

La drenabilidad (CSF) de la pulpa indica que tan rápido puede drenar el agua a través de una fibra. La Figura 6 muestra el análisis de varianza ANOVA para el CSF para las fibras no tratadas y las fibras obtenidas después del pre-tratamiento alcalino. En la Figura 6 se observa que todos los pre-tratamientos disminuyen la drenabilidad de la fibra lo que es un comportamiento opuesto a la retención de agua. Es bien sabido que los pre-tratamientos alcalinos pueden aumentar la fracción de finos en las muestras; estos finos se presentan por el desprendimiento de la superficie, el desenrollado de las fibras o la fractura de las fibras. Los finos producidos retienen más agua y cierran la textura del colchón fibroso ocasionando un drenado más lento [22].

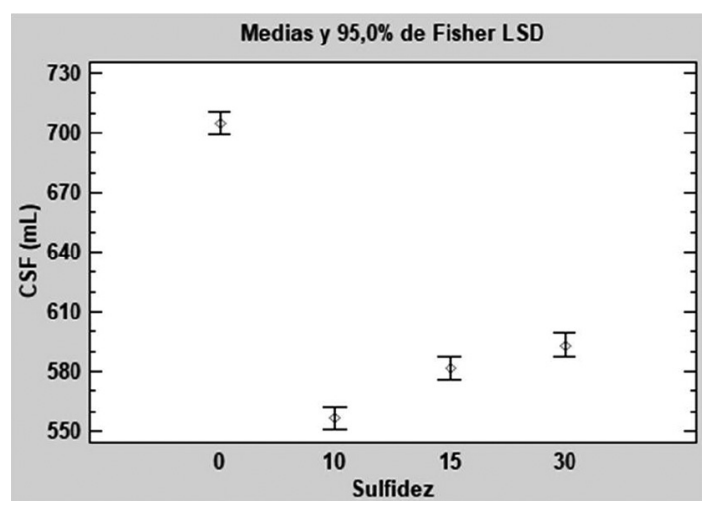

Figura 6. Análisis de Medias e Intervalos LSD para el CSF con relación a la sulfidez del pretratamiento alcalino en fibras de G. angustifolia Kunth.

La retención de agua y la drenabilidad son fenómenos inversos, según los resultados del Análisis de Medias e Intervalos LSD el valor de retención de agua no tiene diferencia estadísticamente significativa con respecto a la sulfidez (Figura 4); sin embargo, drenabilidad muestra una diferencia significativa estadísticamente (Figura 5). Las diferencias estadísticas presentadas en la drenabilidad y en la retención de agua pueden deberse a que la retención de agua es un fenómeno microscópico que indica el agua retenida en la parte interna de la fibra, mientras que la drenabilidad se refiere a un fenómeno macroscópico donde el agua atraviesa un colchón fibroso. Es decir, es un fenómeno que se asocia más a la superficie de la fibra la cual se modifica como se observó en las micrografías SEM.

\section{Respuesta mecánica a 7, 28 y 180 días de fraguado} La relación de agua cemento después de la fabricación de los tableros es un valor que podría influenciar la respuesta a flexión de los tableros de fibrocemento. La Tabla 2 muestra el valor de W/C para los tableros de fibrocemento de acuerdo a las fibras utilizadas para su refuerzo.

Tabla 2. Valores de relación agua - cemento para los tableros compuesto de cemento reforzados con fibras pre-tratadas alcalinamente.

\begin{tabular}{|l|c|}
\cline { 2 - 2 } \multicolumn{1}{c|}{} & Promedio razón de W/C \\
\hline KA10S10 & 0,22 \\
\hline KA10S15 & 0,28 \\
\hline KA10S30 & 0,31 \\
\hline
\end{tabular}


Para realizar una comparación de la respuesta mecánica a los 7, 28 y 180 días de fraguado, se seleccionó un tablero de los tres manufacturados en cada set con un valor de WRV igual o similar. La Figura 7 muestra el cambio de la resistencia a la flexión en los especímenes seleccionados con un tiempo de fraguado de 7, 28 y 180 días. Los valores obtenidos son similares a los obtenidos en otro estudio de fibrocemento donde el refuerzo utilizado también fueron pulpas Kraft de bambú [23].

Se puede observar un incremento del valor de resistencia a la flexión de todos los tableros de cemento reforzados con fibras pre-tratadas comparada con la de los tableros reforzados con fibras no tratadas. Este incremento indica que el pre-tratamiento alcalino mejora la adherencia de las fibras a la matriz de cemento, esta adherencia está relacionada a la remoción de material no celulósico, y la exposición de las fibrillas de celulosa lo que permite un mejor anclaje mecánico. Este comportamiento está en concordancia con los resultados observados mediante las micrografías MEB donde la modificación superficial de la fibra

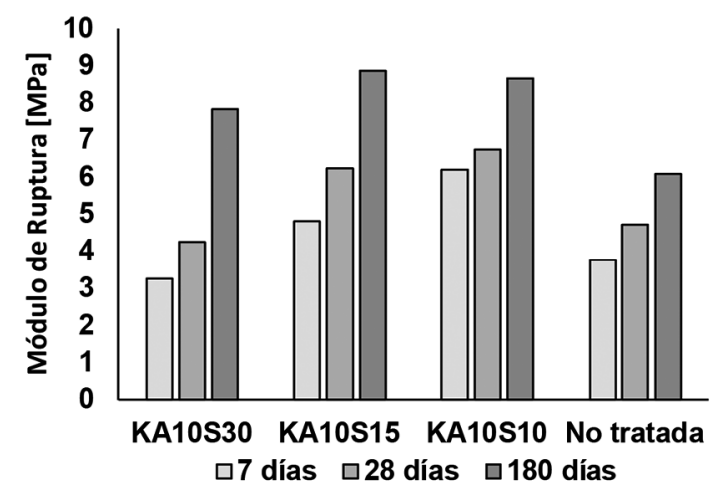

Figura 7. Valores de resistencia a la flexión en función del tiempo de fraguado para tableros de cemento reforzados con fibra de $G$. angustifolia Kunth con valores similares de relación agua/cemento. $($ KA10S30 $=$ Tableros reforzados con fibras tratadas con $30 \%$ de sulfidez; KA10S15 = Tableros reforzados con fibras tratadas con $15 \%$ de sulfidez; KA10S10 = Tableros reforzados con fibras tratadas con $10 \%$ de sulfidez No tratada $=$ tableros reforzados con fibras sin tratamiento). fue evidente después del pre-tratamiento alcalino. En todos los tableros evaluados; la resistencia a la flexión es mayor a los 28 días y no se reduce después de 180 días de fraguado, esto indica que la naturaleza alcalina del cemento permite que se sigan removiendo holocelulosa y lignina, pero no alcanza a degradar de forma significativa la celulosa.

\section{Análisis de Covarianza}

En la Tabla 3 se presentan los resultados del análisis estadístico para tableros compuestos de cemento reforzados con fibras de G. angustfolia Kunth pretratadas alcalinamente. Del análisis puede observarse que solo tienen influencia estadística significativa en la respuesta a la flexión la variación en la sulfidez del pretratamiento y el tiempo de curado ( $\mathrm{p}<0,05)$. Por otro lado, de acuerdo a los resultados obtenidos es posible observar que el W/C no tiene una influencia estadística significativa en la respuesta a la flexión.

El efecto de la relación W/C se ha descrito para concretos y hormigones y se ha reportado un efecto en la respuesta a flexión con relaciones W/C por encima de 0,3 [24]. En este set de resultados los valores medidos de la relación W/C fluctúan entre 0,22 y 0,31 y basados en los resultados encontrados esta relación no afecta los resultados de la respuesta a flexión de los tableros.

Del análisis estadístico (Tabla 3) también se puede observar que no existe interacción entre la sulfidez y el tiempo de fraguado de los tableros ( $p>0,05)$, y esto puede ser atribuido al porcentaje de sustitución de fibras que es solo de unos 5\%. Al no ser una gran masa de sustitución, los cambios químicos y superficiales no modifican el proceso de fraguado del cemento provocando que la cinética sea igual en todos los especímenes. La Figura 8 muestra el resultado del Análisis de Medias e Intervalos LSD del tiempo de fraguado sobre la resistencia a la flexión.

En la Figura 6, se puede observar que los intervalos de confianza no se traslapan, lo que indica que existe una diferencia estadísticamente significativa en la respuesta a flexión con respecto a los días de fraguado. Esto se debe a que a medida que aumenta el tiempo de fraguado el agua retenida por las fibras se libera y permite el curado de las partículas de cemento dentro de la matriz [25]. Mediante el curado interno, la contracción autógena puede prevenirse 
Tabla 3. Análisis estadístico para la respuesta en flexión de tableros compuestos reforzados con fibras de G. angustifolia Kunth.

\begin{tabular}{|l|c|r|c|c|c|}
\hline \multicolumn{7}{|c|}{ Fuente } & Suma de Cuadrados & Gl & Cuadrado Medio & Razón-F* & Valor-P \\
\hline \multicolumn{7}{|c|}{ Covariables } \\
\hline Relación agua/cemento-W/C & 0,337429 & 1 & 0,337429 & 0,69 & 0,4136 \\
\hline Efectos Principales & 13,8627 & 3 & 4,6209 & 9,50 & 0,0003 \\
\hline A = Sulfidez de pretratamiento & 78,793 & 2 & 39,3965 & 80,96 & 0,0000 \\
\hline B = Días de curado & 6,39895 & 6 & 1,06649 & 2,19 & 0,0811 \\
\hline \multicolumn{7}{|c|}{ Interacciones } \\
\hline AB & 11,1928 & 23 & 0,486645 & & \\
\hline RESIDUOS & 110,941 & 35 & & & \\
\hline Total (Corregido)
\end{tabular}

* Todas las razones-F se basan en el cuadrado medio del error residual $\mathrm{P}<0,05$ indica diferencia significativa y $\mathrm{P}>0,05$ no existe diferencia significativa.

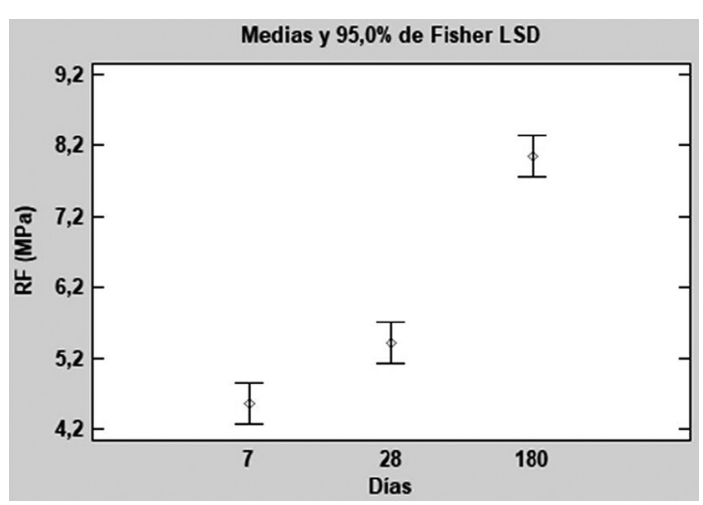

Figura 8. Análisis de Medias e Intervalos LSD de la influencia de los días de curado en la resistencia a la flexión (RF) en tableros compuestos de cemento reforzados con fibras de Guadua angustifolia Kunth.

y la hidratación puede maximizarse lo que genera una mayor compactación y a su vez un aumento en la resistencia a la flexión [26].

La Figura 9 muestra el resultado del Análisis de Medias e Intervalos LSD de la sulfidez sobre la resistencia a la flexión. Como puede observarse existe un valor máximo para la prueba con valores de sulfidez de $10 \%$ y $15 \%$. De la Figura 9 se puede observar que a valores de sulfidez de $30 \%$ la resistencia a la flexión disminuye, y esto puede deberse a que la degradación que sufre la fibra durante el pre-tratamiento alcalino, la exposición de las fibras a altos contenidos de sulfidez puede

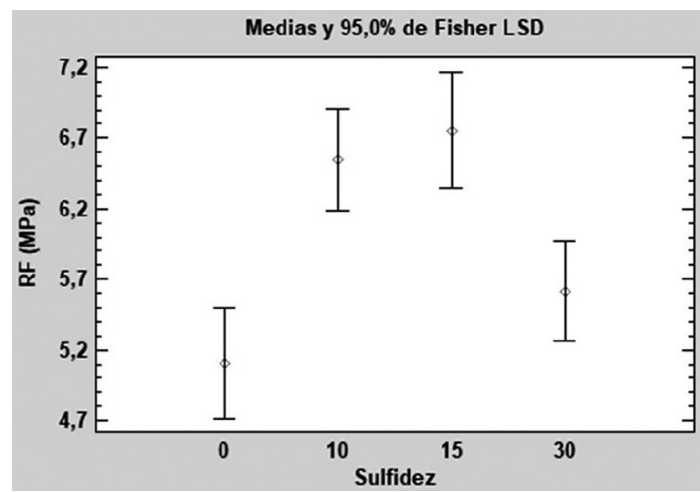

Figura 9. Análisis de Medias e Intervalos LSD de la influencia de la sulfidez de los tratamientos alcalinos en la resistencia a la flexión (RF) en tableros compuestos de cemento reforzados con fibras de Guadua angustifolia Kunth.

afectar la pared celular y en consecuencia se genera una disminución sus propiedades mecánicas [17], $\mathrm{y}$, por lo tanto, se refleja en un decrecimiento de las propiedades de los tableros compuestos de cemento.

\section{Caracterización de la superficie de fractura}

Después de los ensayos de flexión a los 7 y 28 días de fraguado se tomaron imágenes MEB con magnificaciones entre X100 y X200 a los tableros compuestos de cemento con fibras tratadas alcalinamente y tableros con fibras sin tratamiento. La Figura 8 muestra las micrografías de la superficie de fractura de los especímenes después del ensayo de flexión. 
En la Figura 10 es posible observar que en todas las muestras reforzadas con fibras pre-tratadas alcalinamente, el mecanismo de falla predominante de la fibra está asociado a una extracción (pull out) la cual se señala en las imágenes con flechas blancas. Sin embargo, es posible ver que en las muestras de control (fibras sin tratamiento alcalino) el mecanismo de falla predominante es la fractura de la fibra cerca de la matriz de cemento. Esto sugiere que las fibras no tratadas son más frágiles que las obtenidas después de los pre-tratamientos alcalinos. Este comportamiento se relaciona con la respuesta a flexión de los tableros compuestos de cemento previamente encontrada, donde la resistencia a la flexión más baja se encuentra en las muestras de control (refuerzo de fibras no tratadas). Además, es

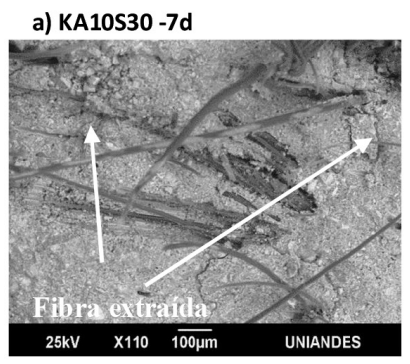

c) KA10S15 -

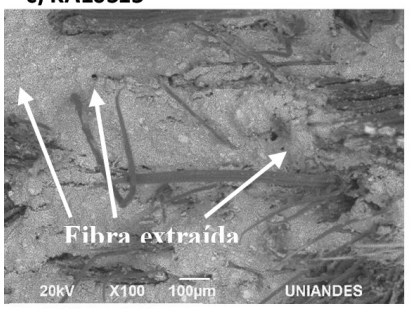

e) KA10S10 -
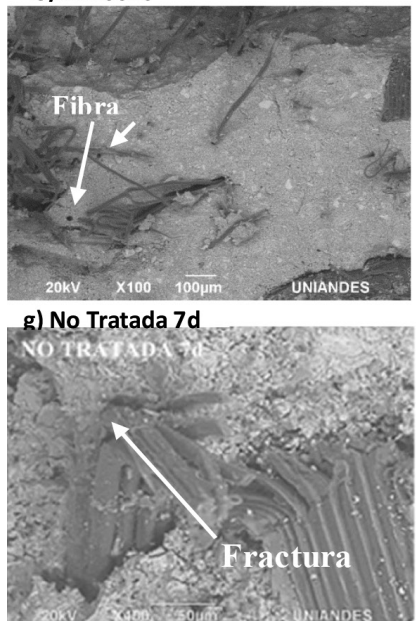

b) KA10\$30 -
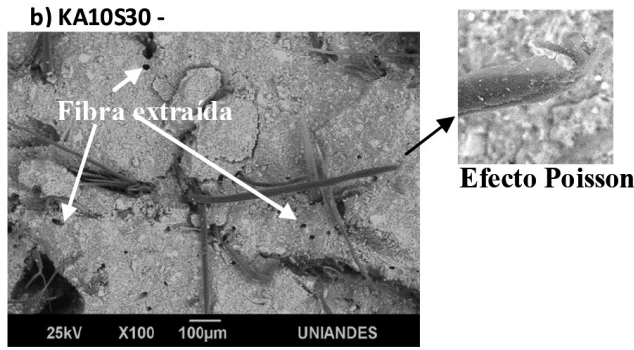

d) KA10S15-28d
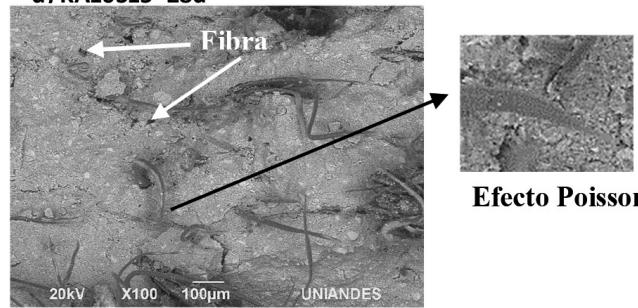

Efecto Poisson
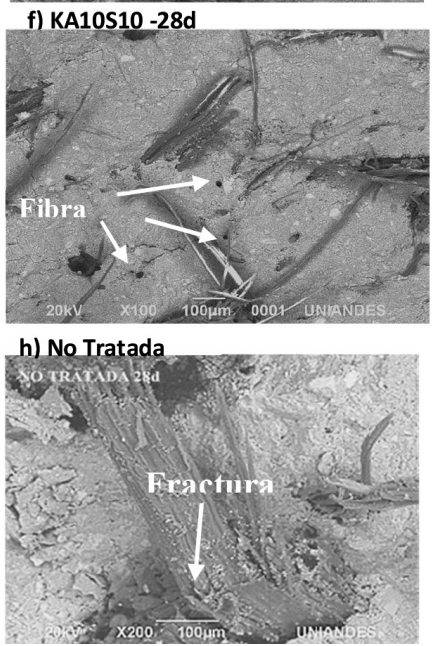

Figura 10.Superficie de fractura de tableros compuestos de cemento reforzados con fibra de Guadua angustifolia Kunth pre-tratadas alcalinamente: $\boldsymbol{a}$-b) Tableros reforzados con fibras tratadas con $30 \%$ de sulfidez a 7 y 28 días de fraguado respectivamente; $\boldsymbol{c}$ - $\boldsymbol{d}$ ) Tableros reforzados con fibras tratadas con $15 \%$ de sulfidez a 7 y 28 días de fraguado respectivamente; $\boldsymbol{e}-\boldsymbol{f}$ ) Tableros reforzados con fibras tratadas con $10 \%$ de sulfidez a 7 y 28 días de fraguado respectivamente; y $\boldsymbol{g}$ - $\boldsymbol{h}$ ) Tableros reforzados con fibras no tratadas a 7 y 28 días de fraguado respectivamente. 
posible notar a los 28 días de curado en las fibras que han sido pre-tratadas alcalinamente un efecto "necking" asociado al efecto Poisson cerca de la región de la falla de las fibras; mientras que las fibras no tratadas muestran un comportamiento frágil que se representa en el rompimiento de las mismas. Este efecto se presenta con el estrechamiento de la fibra cuando se estira longitudinalmente y puede relacionarse con la disipación de energía durante la fractura. Este efecto ha sido reportado en otros estudios donde se ha utilizado la pulpa Kraft para el refuerzo de materiales compuestos [18].

\section{CONCLUSIONES}

El pre-tratamiento alcalino utilizado en las fibras de Guadua angustifolia Kunth permite obtener fibras con superficies más rugosas, eliminando impurezas y materiales no celulósicos. Esta modificación mejora la afinidad en la matriz de cemento. De acuerdo a los resultados encontrados, los menores porcentajes de sulfidez (10\%-15\%) generan fibras más compatibles con la matriz de cemento. La modificación de la superficie de las fibras de G. angustifolia Kunth también modifica el valor de retención de agua y la drenabilidad, convirtiéndolas en fibras más higroscópicas. La liberación del agua retenida por las fibras durante el fraguado facilita el curado interno y previene las contracciones autógenas.

El pre-tratamiento alcalino de las fibras aumenta la resistencia a la flexión de los tableros de cemento alrededor del $50 \%$ a los 180 días de fraguado comparado con tableros fabricados con fibras no tratadas. Los tableros reforzados con fibras KA30S10 y KA30S15 exhiben mayor resistencia mecánica; sin embargo, la diferencia entre estas dos respuestas no es significativa. El pre-tratamiento con $10 \%$ se recomienda como el más adecuado en razón al costo total del pre-tratamiento.

\section{AGRADECIMIENTOS}

Luz Adriana Sánchez agradece a COLCIENCIAS convocatoria 617 la financiación de sus estudios doctorales.

\section{REFERENCIAS}

[1] S.N. Monteiro, F.P.D. Lopes, A.P. Barbosa, A.B. Bevitori, I.L.A. Silva and L. Da Costa. "Natural Lignocellulosic Fibers as Engineering Materials-An Overview". Metallurgical and Materials Transactions A. Vol. $42 \mathrm{~N}^{\circ}$ 10, pp. 2963-2974. 2011.

[2] H. Savastano Jr., P.G. Warden and R.S.P. Coutts. "Performance of low-cost vegetable fibre-cement composites under weathering". CIB World Building Congress. Wellington, New Zealand, pp. 1-11. April 2001.

[3] M. Ardanuy, J. Claramunt, R.D. Toledo Filho. "Cellulosic fiber reinforced cement-based composites: A review of recent research". Construction and Building Materials Vol. 79, pp. 115-128. 2015.

[4] R.D. Tolêdo Filho, K. Scrivener, G.L. England, and K. Ghavami. "Durability of alkali-sensitive sisal and coconut fibres in cement mortar composites". Cement and Concrete Composites. Vol. 22, Issue 2, pp. 127-143. 2000.

[5] J.M. Ferraz, C.H.S. del Menezzi, D.E. Teixeira and S.A. Martins. "Effects of treatment of coir fiber and cement/fiber ratio on properties of cement-bonded composites". BioResources. Vol. $6 \mathrm{~N}^{\circ}$ 3, pp. 3481-3492. 2011.

[6] J. Wei and C. Meyer. "Improving degradation resistance of sisal fiber in concrete through fiber surface treatment". Applied Surface Science. Vol. 289, pp. 511-523. 2014.

[7] R.D. Tolêdo Filho, K. Ghavami, G.L. England, and K. Scrivener. "Development of vegetable fibre-mortar composites of improved durability". Cement and Concrete Composites. Vol. $25 \mathrm{~N}^{\circ}$ 2, pp. 185-196. 2003.

[8] S. Chakraborty, S.P. Kundu, A. Roy, B. Adhikari, and S.B. Majumder. "Effect of jute as fiber reinforcement controlling the hydration characteristics of cement matrix". Industrial and Engineering Chemistry Research. Vol. 52 NN $^{\circ}$ 3, pp. 1252-1260. 2013.

[9] G.H.D Tonoli, M.N. Belgacem, G. Siqueira, J. Bras, H. Savastano, and F.A. Rocco Lahr. "Processing and dimensional changes of cement based composites reinforced with surface-treated cellulose fibres". Cement and Concrete Composites. Vol. $37 \mathrm{~N}^{\circ} 1$, pp. 68-75. 2013. 
[10] J. Wei and C. Meyer. "Degradation mechanisms of natural fiber in the matrix of cement composites". Cement and Concrete Research. Vol. 73, pp. 1-16. 2015.

[11] M. Cai, H. Takagi, A.N. Nakagaito, Y. Li, and G.I.N. Waterhouse. "Effect of alkali treatment on interfacial bonding in abaca fiber-reinforced composites". Composites Part A. Vol. 90, pp. 589-597. 2016.

[12] M. Khorami, E. Ganjian, A. Mortazavi, S. Messaoud, A. Olubanwo, and A. Gand. "Utilisation of waste cardboard and Nano silica fume in the production of fibre cement board reinforced by glass fibres". Construction and Building Materials. Vol. 152, pp. 746-755. 2017.

[13] C.L. Pereira, H. Savastano, J. Payá, S.F. Santos, M.V. Borrachero, J. Monzó, and L. Soriano. "Use of highly reactive rice husk ash in the production of cement matrix reinforced with green coconut fiber". Industrial Crops and Products. Vol. 49, pp. 88-96. 2013.

[14] S. Velasquez, J. Monzó, M. Borrachero, L. Soriano, and J. Payá. "Evaluation of the pozzolanic activity of spent FCC catalyst/ fly ash mixtures in Portland cement pastes". Thermochimica Acta. Vol. 632, pp. 29-36. 2016.

[15] Z. Yuan, N.S. Kapu, R. Beatson, D.M. Martinez, and X. Feng. "Effect of alkaline pre - extraction of silica and hemicellulose on kraft pulping of bamboo". Ind. Crop. Prod. Vol. 91, pp. 1-41. 2016.

[16] K. Heise, C. Rossberg, J. Strätz, C. Bäurich, E. Brendler, H. Keller, and S. Fischer. "Impact of pre-treatments on properties of lignocelluloses and their accessibility for a subsequent carboxymethylation". Carbohydrate Polymers. Vol. 161, pp. 82-89. 2017.

[17] M. Ek, G. Gellerstedt, and G. Henriksson. Pulping Chemistry and Technology Vol 2. Ed. Deutsche National bibliothek. 2009.
[18] B.J. Mohr, H. Nanko, and K.E. Kurtis. "Durability of kraft pulp fiber - cement composites to wet / dry cycling". Cement and Concrete Composites. Vol. 27, pp. 435448. 2005.

[19] C.J. Biermann. "Handbook of Pulping and Papermaking". In Academic Press (Ed.), p. 741. London: Elsevier. 1996.

[20] D. Montgomery and G. Runger. "Applied statistics and probability for engineers". 5th ed. Hoboken, NJ: Wiley. 2011.

[21] R. Ponni, L. Galvis, and T. Vourinen. "Changes in accessibility of cellulose during kraft pulping of wood in deuterium oxide". Carbohydrate Polymers. Vol. 101, pp. 792797. 2014.

[22] T. Escoto. "Evaluación de la calidad en pulpa, papel y cartón". Universidad de GuadalajaraDepartamento de Madera Celulosa y Papel. Las Agujas Zapopan, Jal, México. 2004.

[23] M. Khorami, E. Ganjian, and A. Sirvastavb. "Feasibility Study on Production of Fiber Cement Board Using Waste Kraft Pulp in Corporation with Polypropylene and Acrylic Fibers". Materials Today: Proceedings. Vol. 3, pp. 376-380. 2016.

[24] I. Tunde, Y. Alaro, and W. Adebayo. "An appropriate relationship between flexural strength and compressive strength of palm kernel shell concrete". Alexandria Engineering Journal. Vol. 55; pp. 1553-1562. 2016.

[25] D. Snoeck, L. Pel, and N. De Belie. "The water kinetics of superabsorbent polymers during cement hydration and internal curing visualized and studied by NMR". NATUREScientific Reports. Vol. 7 No 9514, pp. 1-14. 2017.

[26] D.P. Bentz, and O.M. Jensen. "Mitigation strategies for autogenous shrinkage cracking". Cement and Concrete Composites. Vol. 26, pp. 677-685. 2004. 